\title{
Erratum to: EMBEC \& NBC 2017
}

Hannu Eskola, Outi Väisänen, Jari Viik, and Jari Hyttinen

BioMediTech Institute and Faculty of Biomedical Sciences and Engineering,

Tampere University of Technology, Tampere, Finland

\section{Erratum to:}

H. Eskola et al. (eds.),

EMBEC \& NBC 2017,

IFMBE Proceedings 65,

https://doi.org/10.1007/978-981-10-5122-7

In the original version of the book, the following belated correction have been incorporated: The author name "Carla Iacconi" has been changed to "Chiara Iacconi" in the Frontmatter, Backmatter and in Chapter 110.

The updated online version of this book can be found at https://doi.org/10.1007/978-981-10-5122-7

(C) Springer Nature Singapore Pte Ltd. 2018

H. Eskola et al. (eds.), EMBEC \& NBC 2017,

IFMBE Proceedings 65,

https://doi.org/10.1007/978-981-10-5122-7_283 